\title{
Unclear Reason of Recurrent Graft Infections after Aortobifemoral Reconstruction
}

\author{
Srdjan Babic ${ }^{1,2}$, Vuk Jovanovic $^{1 *}$, Milan Marinkovic $^{1}$, Slobodan Tanaskovic ${ }^{1,2}$, Predrag Gajin ${ }^{1,2}$, Nenad Iljevski $^{1,2}$ \\ ${ }^{1}$ Department of Clinic for Vascular Surgery, Institute for Cardiovascular Diseases "Dedinje”, Belgrade, Serbia; ${ }^{2}$ School of \\ Medicine, University of Belgrade, Belgrade, Serbia
}

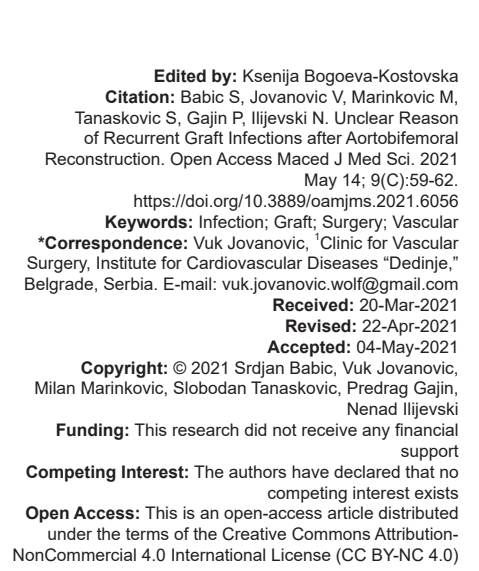

Introduction

Aortic graft infection is one of the most serious complications of vascular reconstruction. According to the literature, the incidence of infection is about $1 \%$, with no difference in the incidence of infection whether open or endovascular graft implantation is performed [1].

Etiologically, the most common causes are bacteria from the external environment that make up the commensal flora of the skin such as Staphylococcus epidermidis [2]. Furthermore, the graft contamination could arise from infectious atheromatous plaques of the aorta [2], colon, and small intestine fistulas or erosion [3], [4], and from the urinary tract [5].

Thegraftinfection often presentsas earlyinfection in time period of 3 months after surgery, commonly with local signs, fever and elevated inflammatory markers, or months or years after surgery, manifested as fever, malaise, weight loss, and arthralgia [6]. Data from the literature suggest that survival rate for early infections is $20 \%$ and $70 \%$ for late ones [7].

Multidetector computed tomography (MDCT) is the most sensitive diagnostic method for verifying certain changes that accompany graft infection, such as intestinal bleeding, swelling in the inguinal region, or fistulas [7].

We present three cases of late " $Y$ " graft infection with unknown etiology.

\section{Case Report}

\section{Case 1}

A male patient, aged 74, underwent abdominal aorta aneurysm (AAA) resection in 2012 with aortobifemoral reconstruction with Dacron " $Y$ " graft. The post-operative course was uneventful and the patient was discharged in good general condition. After 6 years, the patient was presented with swelling in inguinal region and elevated inflammatory markers, and was admitted due to an abscess in the area of the left iliac fossa with a fistulous canal on the anterior abdominal wall verified by MDCT (Figure 1).

Wound swab detected coliform bacteria and revealed multiple diverticula without signs of inflammation. First, we extracted left side of bifurcated "Y" graft and then performed femorofemoral cross-over 
bypass. Six months later, the patient was admitted again due to recurrent abscess. Repeated MDCT scan could not reveal the original site of infection and the abscess was not in contact with the graft. Evacuation of the abscess was performed. After 2 and 3 years, we performed evacuation of retroperitoneal abscess again.

\section{Case 2}

A 64-year-old male patient was admitted due to fever, swelling, and pain in the left hemiabdomen. He also underwent aortobifemoral revascularization with a "Y" graft 9 years ago. After laboratory examination which showed elevation of inflammatory markers, the MDCT verified retroperitoneal abscess collection with involvement of the left iliopsoas muscle extending to the left pararenal space with graft involvement in the left groin (Figures 2-4). Furthermore, MDCT revealed left colonic diverticula without signs of inflammation. Two sets of blood cultures, as well as sample for coproculture on Clostridium difficile toxin were negative. From urine culture, Enterococcus species was isolated. We performed midline relaparotomy and after detailed exploration of the peritoneal cavity verified the absence of contact between the descending colon, which was partially separated from the posterior wall, and the abscess cavity. A counter incision was made on the lateral part of the anterior abdominal wall, a large amount of purulent contents was drained, wound swab came positive for Candida spp.

\section{Case 3}

The third case was a 66-year-old female patient admitted because of swelling and presence of fistula in the right iliac femoral region. Computed tomography (CT) verified purulent abscess in the previously mentioned region. Six years ago, she underwent AAA

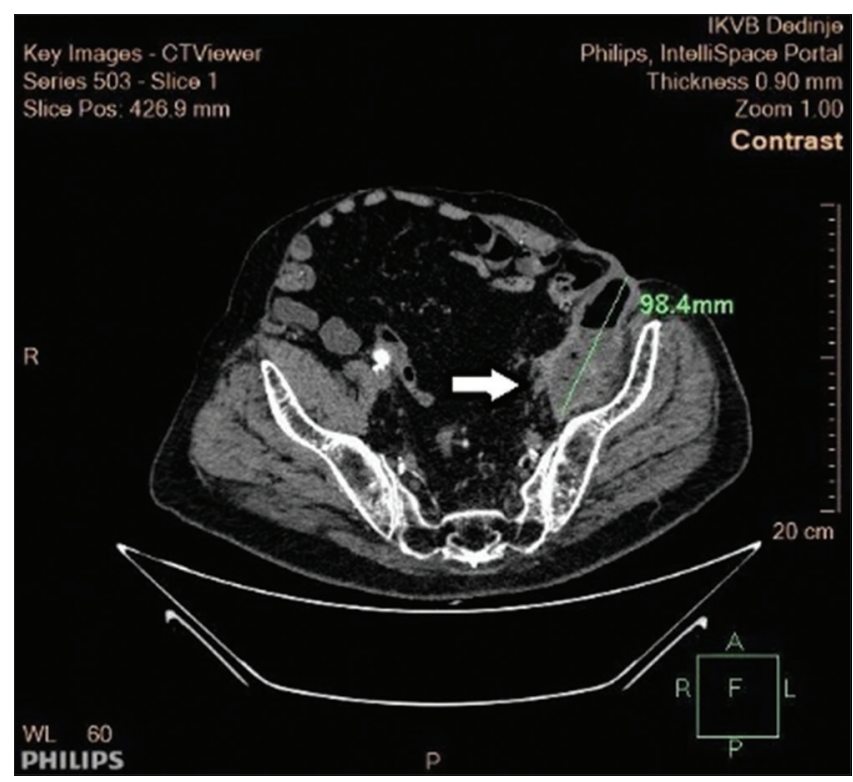

Figure 1: Multidetector computed tomography finding - purulent collection in the left iliac fossa resection with aortobifemoral reconstruction. In the following year, she has been treated several times in another hospital because of hematemesis and melena of unknown origin. Gastroscopy was performed but did not show the source of bleeding at that point.

First, we performed evacuation of the abscess only, because there were no signs of graft infection. After a few months, she was hospitalized again due to high fever and pain at rest in her right leg, when the purulent collection was verified by multislice computed tomography (MSCT) with hydronephrosis of the right kidney. We performed again abscess evacuation and the swab culture came positive for Klebsiella pneumoniae and Enterococcus faecalis. Eight months later, the patient was readmissioned due to MSCT verified purulent collection in the right inguinal region with graft involving (Figure 5).

$A$ reevacuation of the purulent contents was performed with extra-anatomic silver coated graft revascularization. The operation and the post-operative course went well, the patient was discharged in good condition.

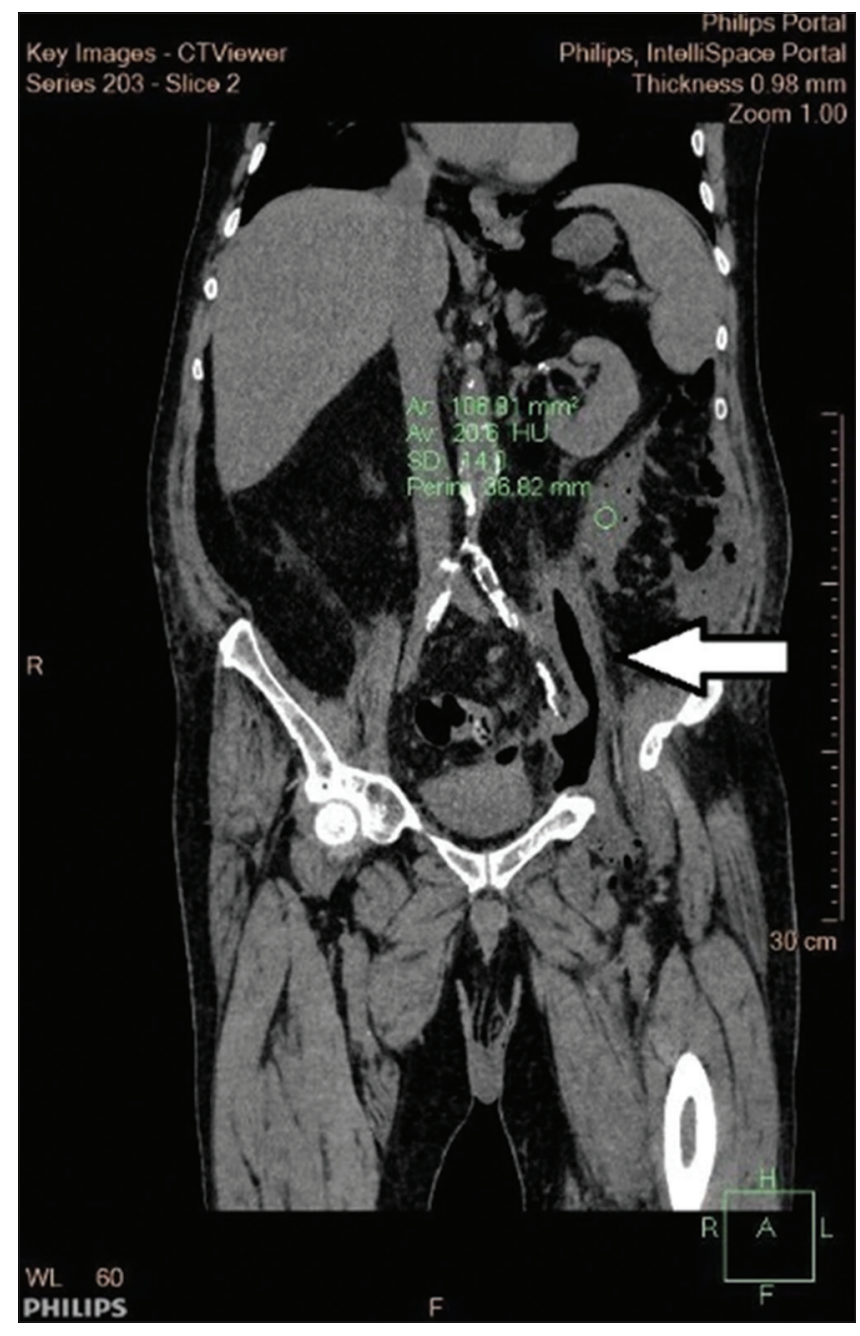

Figure 2: Multidetector computed tomography finding - abscess collection with involvement of the left iliopsoas muscle, colon diverticula 


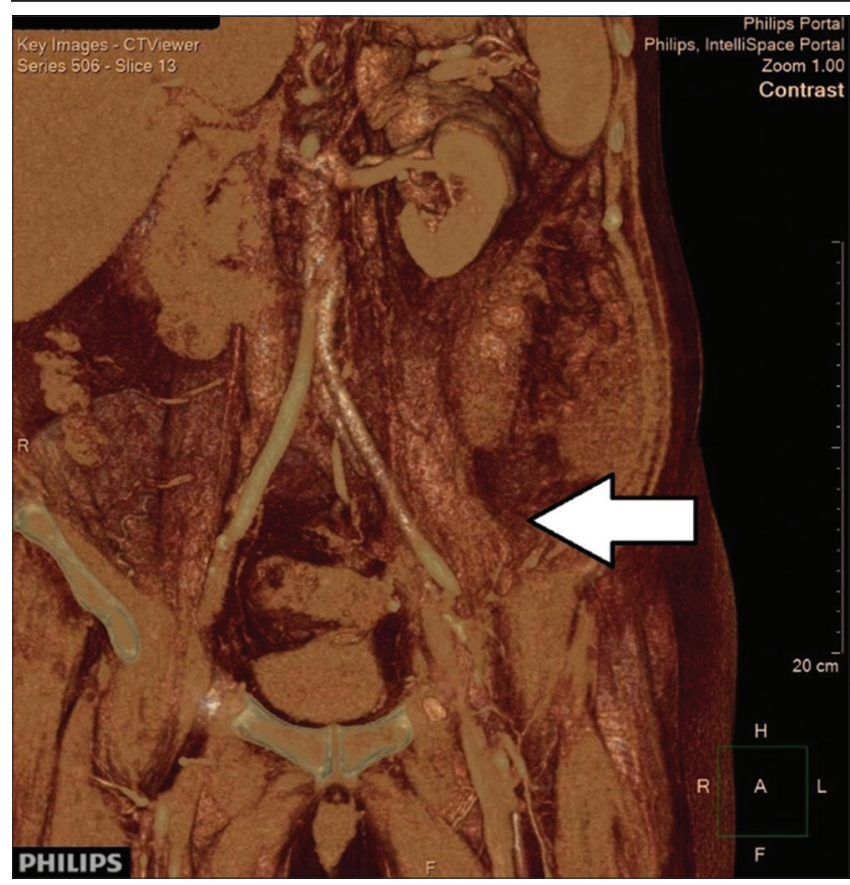

Figure 3: $3 D C T$ finding $-3 D$ reconstruction of abscess collection with involvement of the left iliopsoas muscle

\section{Discussion}

In our study, wound swab came positive in all three cases - in the first one for coliform bacteria, in the second for Candida spp., and in the third case for $K$. pneumoniae and $E$. faecalis. These findings are in accordance to the literature, which suggest that type of pathogen depends on the site of infection - polymicrobial infections were frequently identified in patients with abdominal prosthetic vascular graft

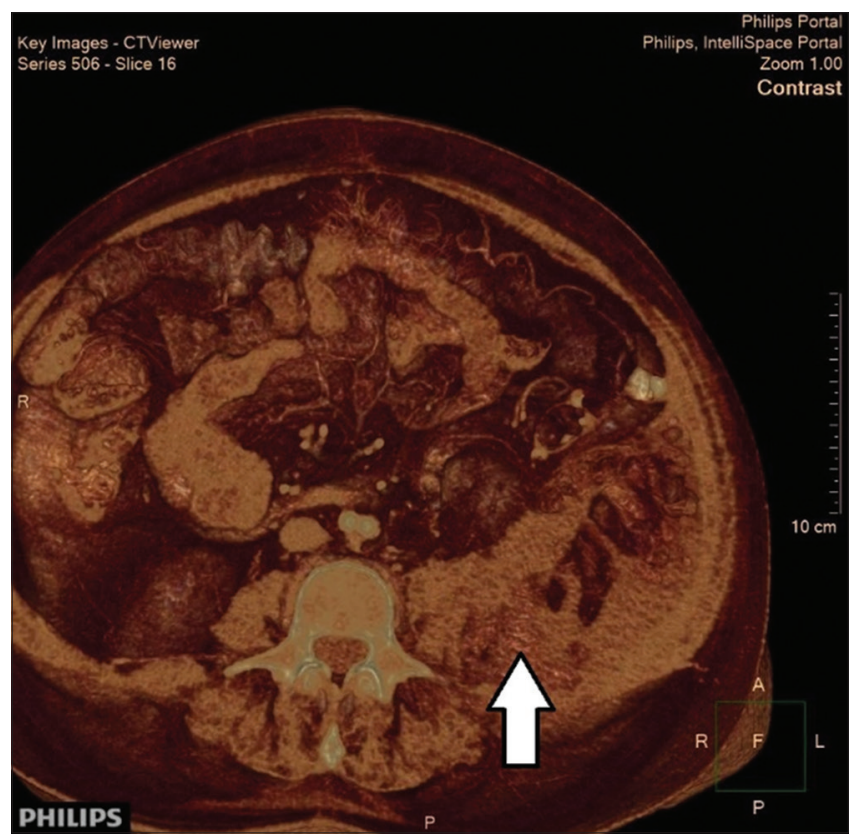

Figure 4: 3D CT finding - purulent collection in the left retroperitoneal space, colon diverticula

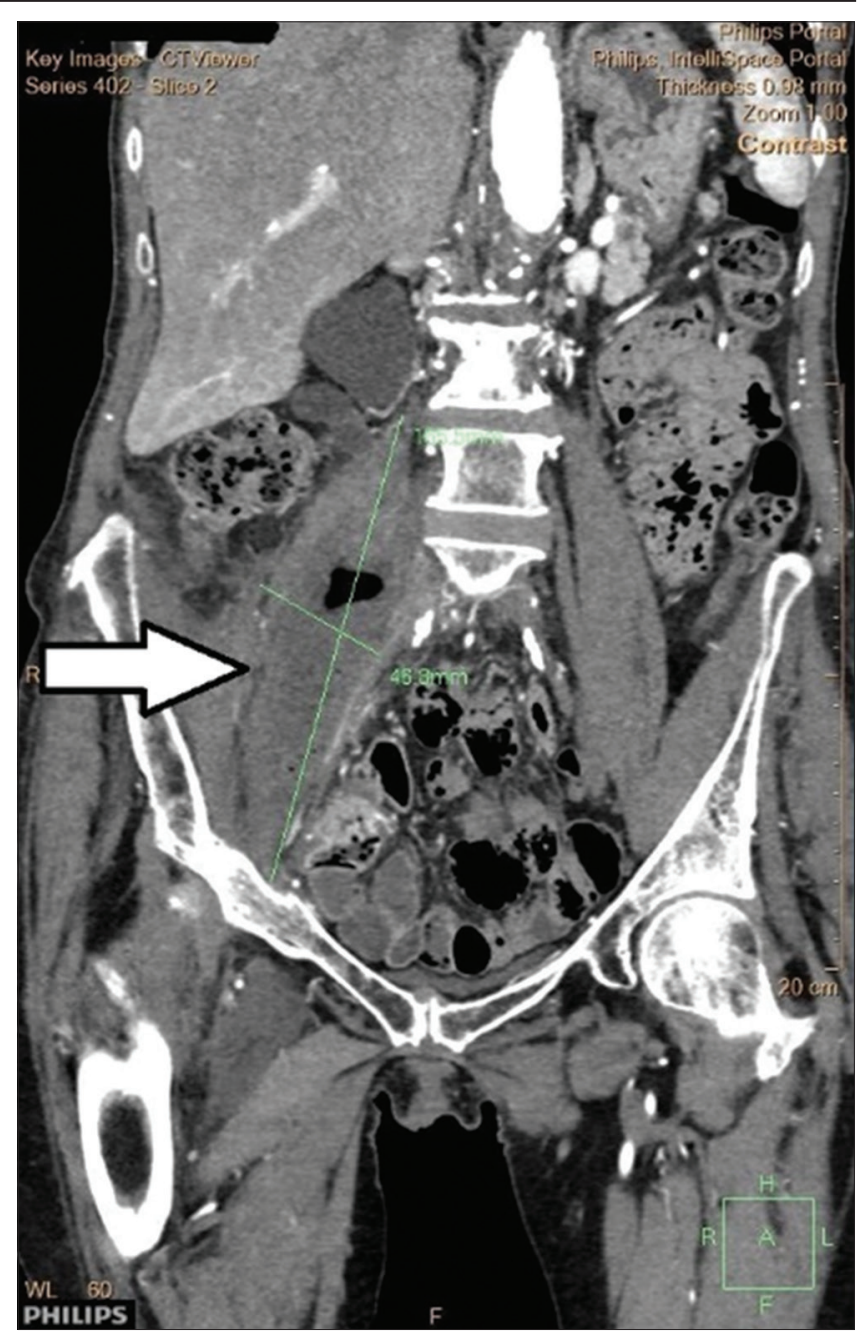

Figure 5: Multidetector computed tomography finding - purulent collection in the right inguinal region, purulent swelling of the right iliopsoas muscle with gas

infection comprising mainly Gram-negative and anaerobic bacteria, enterococci, and Candida spp. [8].

However, we should also have in mind that in the literature, other pathogens have been also described, which are more difficult to detect due to their characteristics, for why it is necessary to take samples of graft and periprosthetic material. Furthermore, this type of pathogen, with a low degree of virulence, shows the ability to create a biofilm, due to which the penetration of antibiotics is difficult, which prolongs the infection and prevents its complete remediation with a conservative approach [9]. These data indicate that there is a possibility that such a pathogenic agent will not be detected in time and that it will lead to a prolongation and flaring-up of the infection for the previously mentioned reasons.

In all three of our patients, the clinical presentation included the presence of swelling in the inguinal region, and in two of them, fistulas, accompanied by fever and elevated markers of inflammation. What is interesting is that two patients had proven intestinal pathology, most commonly diverticula of the left colon. Data from the literature show that graft infections can be the result of erosion of the small and large intestine 
due to graft compression [4], compression necrosis of the colon by graft action [10], as well as iatrogenic graft malposition [5].

Given the bacteriological findings, imaging techniques showed the presence of old changes in the sigmoid colon, and the recurrent course of infection, we suspect that the cause of infection in the first case was due to gastrointestinal pathology.

In the second case, during the relaparotomy, the partial separation of the descending colon from the posterior wall was verified, which may suggest that the infection did occur due to events in the gastrointestinal tract, but diverticula presence could also be a possible cause.

In the third case, a female patient had medical history of previous gastrointestinal bleeding. We suspected on gastrointestinal etiology, although source of bleeding was not found during gastroscopy. Data from the literature show that late graft infections are manifested as enteral bleeding in $31 \%$ of cases, where CT was used to verify bleeding in $94 \%$ of cases, while endoscopy diagnosed only in slightly more than half of the cases, that is, $53 \%$ [7]. This data suggest that, to make a faster and safer diagnosis and prevent further complications, in addition to endoscopy, it may be necessary to perform a CT examination, in cases when there is report of hematemesis or melena and the results of endoscopy do not suggest source of bleeding [7]. In that case, the introduction of CT examination as part of the diagnostic algorithm in the mentioned situations should be considered.

In all three cases, we performed in situ surgery and extra-anatomic bypass, which is in accordance with the vascular guidelines [11]. Given the fact that it did not provide expected results because it did not prevent the occurrence of recurrent infections, the question arises whether aggressive surgical treatment, as for example, Hartmann procedure, could have prevented future graft infections and so be a possible treatment of choice in similar cases.

\section{Conclusion}

Graft infections after aortobifemoral reconstruction carry the risk of frequent rehospitalizations due to the inability to completely eradicate the infection. The medical histories of our patients in the review indicate that detecting the mechanisms of infection is not an easy process, and despite undertaking diagnostic procedures that are the gold standard and therapeutic protocols, frequently the etiology remains unclear. Further studies should be focused on events in gastrointestinal tract as possible source of graft infection and investigate if some aggressive treatments, for example, Hartmann procedure could prevent development of future graft infection.

\section{References}

1. Shiraev T, Barrett S, Heywood S, Mirza W, Hunter-Dickson M, Bradshaw C, et al. Incidence, management, and outcomes of aortic graft infection. Ann Vasc Surg. 2019;59:73-83. https://doi. org/10.1016/j.avsg.2019.01.027

PMid:31009718

2. Schurink GW, Peppelenbosch N, Mees B, Jacobs MJ Diagnostic algorithms and treatment strategies in primary aortic and aortic graft infections. J Cardiovasc Surg (Torino). 2016;57(2):224-32.

PMid:26745263

3. Saito H, Nishikawa Y, Akahira Jl, Yamaoka H, Okuzono T, Sawano $\mathrm{T}$, et al. Secondary aortoenteric fistula possibly associated with continuous physical stimulation: A case report and review of the literature. J Med Case Rep. 2019;13(1):61. https://doi.org/10.1186/s13256-019-2003-1

PMid:30871625

4. Uslu HY, Kurt H. A case report of a unique aorto-bifemora graft infection and its treatment. J Surg Case Rep. 2020;2020(11):rjaa382. https://doi.org/10.1093/jscr/rjaa382 PMid:33214865

5. Blank JJ, Rothstein AE, Lee CJ, Malinowski MJ, Lewis BD, Ridolfy $\mathrm{TJ}$, et al. Aortic graft infection secondary to iatrogenic transcolonic graft malposition. Vasc Endovascular Surg. 2018;52(5):386-90. https://doi. org/10.1177/1538574418764037

PMid:29554857

6. Chapman AS, Delgadillo D, MacGuidwin E, Greenberg JI, Jameson AP. Graft infection masquerading as rheumatologic disease: A rare case of aortobifemoral graft infection presenting as hypertrophic osteoarthropathy. Ann Vasc Surg. 2017;41:283. e11-8. https://doi.org/10.1016/j.avsg.2016.10.041 PMid:28279724

7. Soetevent C, Klemm PL, Stalenhoef AF, Bredie SJ. Vascular graft infection in aortoiliac and aortofemoral bypass surgery: Clinical presentation, diagnostic strategies and results of surgical treatment. Neth J Med. 2004;62(11):446-52. PMid:15685895

8. Erb S, Sidler JA, Elzi L, Gurke L, Battegay M, Widmer AF, et al. Surgical and antimicrobial treatment of prosthetic vascular graft infections at different surgical sites: A retrospective study of treatment outcomes. PLoS One. 2014;9(11):e112947. https:// doi.org/10.1371/journal.pone.0112947

PMid:25393400

9. Pallotto C, Simonte G, Verzini F, Lenti M, Baldelli $F$, Francisci D, et al. Early abdominal aortic graft infection due to Propionibacterium acnes: A case report and review of the literature. J Chemother. 2018;30(3):185-8. https://doi.org/10.10 80/1120009x.2018.1451453

PMid:29557272

10. Okadome K, Watanabe T, Kina M, Kusaba A, Inokuchi K. Skin sliding closure technique is effective for management of infected prostheses in cases of arterial reconstruction with synthetic grafts. Jpn J Surg. 1982;12(5):349-55. https://doi.org/10.1007/ bf02469634

PMid:6755009

11. Chaikof EL, Dalman RL, Eskandari MK, Jackson BM, Lee AW, Mansour AM, et al. The society for vascular surgery practice guidelines on the care of patients with an abdominal aortic aneurysm. J Vasc Surg. 2018;67(1):2-77. https://doi. org/10.1016/j.jvs.2017.10.044

PMid:29268916 